\title{
DUALITY FOR THE LEVEL SUM OF QUASICONVEX FUNCTIONS AND APPLICATIONS
}

\author{
M. VOLLE
}

\begin{abstract}
We study a quasiconvex conjugation that transforms the level sum of functions into the pointwise sum of their conjugates and derive new duality results for the minimization of the max of two quasiconvex functions. Following Barron and al., we show that the level sum provides quasiconvex viscosity solutions for Hamilton-Jacobi equations in which the initial condition is a general continuous quasiconvex function which is not necessarily Lipschitz or bounded.
\end{abstract}

\section{INTRODUCTION}

The powerful properties of the Legendre-Fenchel conjugation for convex functions can be summarized by saying that, under some mild assumptions (also called qualification conditions), it exchanges the pointwise sum of functions with the infimal convolution of their conjugates and vice versa $([15]$, [27]). The geometrical interpretation of the infimal convolution of two extended real-valued functions is well known: it lies in the fact that the vectorial (or Minkowski) sum of the strict epigraphs of two extended real-valued functions is nothing but the strict epigraph of their infimal convolution. By the way such a property is at the origin of another terminology in which the infimal convolution is called epigraphical sum ([2] [3]). When dealing with quasiconvex functions this operation suffers from the fact that the epigraphical sum of two quasiconvex functions is no longer quasiconvex ([18]). However, there exists an interesting substitute for the epigraphical sum in the field of quasiconvexity, namely, the level sum of functions, that is the function whose strict lower level sets are the vectorial sum of the strict lower level sets of the initial functions ([18] [28] [30] [33],...). Thus the level sum of two quasiconvex functions is still quasiconvex. Another way to introduce the level sum is obtained by perturbing the problem of minimizing the max of two extended real-valued quasiconvex functions. It appears that such a problem plays an analogous role to the one played by the minimization of the sum of two convex functions in convex optimization ([35]). Moreover the level sum has also been recently used to obtain a viscosity solution of the Hamilton-Jacobi equation $u_{t}+H(D u, u)=0$ arising in the minimization of the maximum cost in $L^{\infty}$ spaces; here the Hamiltonian $H$ is sublinear in the first variable and nondecreasing in the second variable $([7],[8])$. In this paper we introduce and study a conjugation for quasiconvex functions,

University of Avignon, Department of Mathematics, 33, rue Louis Pasteur, 84000 Avignon, France. E-mail: michel.volle@univ-avignon.fr.

Received by the journal September 2, 1997. Revised May 7, 1998. Accepted for publication May 15, 1998.

(C) Société de Mathématiques Appliquées et Industrielles. Typeset by LATEX. 
slightly different from those introduced by Crouzeix in [11] [12] and Barron and Liu in [8], that transforms the level sum of arbitrary extended realvalued functions into the pointwise sum of their conjugates. Such a property is not shared by the more or less classical conjugations already introduced in quasiconvex duality theory ([19] [20] [25] [29] [31] [32] [34],...). Another advantage of the biconjugation we present is that it constitutes a Galois correspondence; nevertheless it is not a generalized conjugation in the sense of Moreau ([21]). In fact it enters in the general framework introduced by J.-P. Penot and the author $([23])$ but has never been studied for its own interest. The classes of regular functions related to this biconjugation are entirely characterized in theorems 3.4 and 3.5. The case of radial functions is studied in details in Propositions 4.10, 4.11 and Corollaries 4.12, 4.13. Under a qualification condition involving the directions of majoration of the functions, we prove that the level sum of two quasiconvex lower semicon-

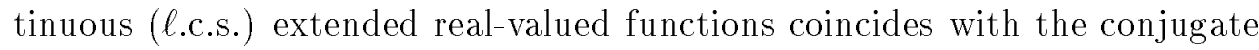
of the sum of their conjugates (Theorem 4.2). As a by-product we get dual problems with zero duality gap for the global minimization of the max of two quasiconvex $\ell$.s.c. extended real-valued functions (Theorem 4.5 and its corollaries). In this way we recapture a dual problem introduced in [35] with different conditions ensuring a zero duality gap. Finally, following Barron and al.([7]), we show that the level sum provides a quasiconvex viscosity solution of the Hamilton-Jacobi equation $u_{t}+H(D u, u)=0, u(0, \cdot)=g$ when the initial condition $g$ is a general continuous quasiconvex function which is not necessarily Lipschitz or bounded (Proposition 5.2, Theorem 5.5), a result announced in [36].

More generally, the present paper may be viewed as a contribution to minimax analysis [5], [16].

\section{DEFinitions, NOTATIONS}

In the sequel $X$ is a Hausdorff locally convex topological vector space with topological dual $Y$ and we denote by $\langle$,$\rangle the bilinear mapping \langle x, y\rangle:=$ $y(x)(x \in X, y \in Y)$. Given an extended real-valued function $f: X \rightarrow \overline{\mathbb{R}}$ and $t \in \overline{\mathbb{R}}:=\mathbb{R} \cup\{-\infty\} \cup\{+\infty\}$ we set

$$
\{f<t\}=\{x \in X: f(x)<t\}
$$

for the strict lower level set (at level $t$ ) of $f$. Observe that $\{f<t\}$ is nonempty iff $t>\inf _{X} f$. The function $f$ is said to be quasiconvex if all its strict lower sets are convex. Of course this amounts to saying that all its lower sets $\{f \leq t\}(t \in \mathbb{R})$ are convex, or that

$$
f(t u+(1-t) v) \leq \max (f(u), f(v)):=f(u) \vee f(v)
$$

for all $u, v \in X, t \in[0,1]([4],[13], \ldots)$.

By adding strict lower sets of two extended real-valued functions $f, g$ : $X \rightarrow \overline{\mathbb{R}}$,

$$
\{f<t\}+\{g<t\}:=\{u+v: u \in\{f<t\}, v \in\{g<t\}\},
$$

one obtains the strict lower level set of the function

$$
x \in X \longmapsto(f \triangle g)(x):=\inf (f(u) \vee g(v): u+v=x) .
$$


More precisely, one has

$$
\{f \triangle g<t\}=\{f<t\}+\{g<t\}
$$

for all $t \in \overline{\mathbb{R}}$. Here we adopt the convention

$$
A+\emptyset=\emptyset+A=\emptyset
$$

for any subset $A \subset X$ accordingly to the relation

$$
\inf _{X}(f \triangle g)=\inf _{X} f \vee \inf _{X} g .
$$

The function $f \triangle g$ is called the level sum of $f$ and $g$. It is quasiconvex whenever the functions $f$ and $g$ are so.

Support functions will play a central role in the paper. Recall that the support function of a subset $A \subset X$ is defined by

$$
y \in Y \longmapsto i_{A}^{*}(y):=\sup _{x \in A}\langle x, y\rangle,
$$

with the convention sup $\emptyset=-\infty$. In other words the support function of $A$ is the Legendre-Fenchel conjugate of the indicator function $i_{A}$ of $A\left(i_{A}(x)=0\right.$ if $x \in A, i_{A}(x)=+\infty$ if $\left.x \in X \backslash A\right) ; i_{A}^{*}$ is positively homogeneous (p.h.) $\left(i_{A}^{*}(\lambda y)=\lambda i_{A}^{*}(y)\right.$ whenever $\left.\lambda>0, y \in Y\right)$ and convex; this means that $i_{A}^{*}$

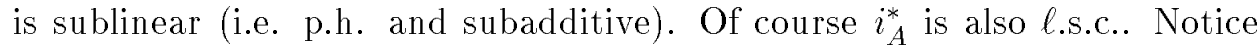
that $i_{A}^{*}$ is either proper (i.e. $i_{A}^{*}(Y) \subset \mathbb{R} \cup\{+\infty\}$ and $i_{A}^{*}(Y) \neq\{+\infty\}$ ) or identically $-\infty$. In fact, Hormander's theorem ([17]) says that the class of support functions coincides with the class of $\ell$.s.c. sublinear extended real-valued functions which are either proper or identically $-\infty$.

Taking 2.3 into account one has (for all $y \in Y$ ),

$$
i_{A+B}^{*}(y)=i_{A}^{*}(y)+i_{B}^{*}(y)
$$

where $(+\infty)+(-\infty)=(-\infty)+(+\infty)=-\infty$.

Of course we set $(+\infty) \dot{+}(-\infty)=(-\infty) \dot{+}(+\infty)=+\infty$. The operations + and $\dot{+}$ enjoy calculus rules $([21])$ : in particular we shall use the fact that, for all extended-real numbers $r, s, t \in \overline{\mathbb{R}}$, the following equivalence holds:

$$
s \dot{+} t<r \Longleftrightarrow \exists s_{1}, t_{1} \in \overline{\mathbb{R}}: r=s_{1} \dot{+} t_{1}, s<s_{1}, t<t_{1} .
$$

\section{A Galois CORRESPONDENCE FOR QUASICONVEX FUNCTIONS}

To each extended real-valued function $f: X \rightarrow \overline{\mathbb{R}}$ let us associate the conjugate function $f^{c}$ defined by

$$
f^{c}(y, r):=\sup _{f(x)<r}\langle x, y\rangle=\sup _{\{f<r\}}\langle\cdot, y\rangle
$$

for all $(y, r) \in Y \times \mathbb{R}$. Let us first observe that this conjugation is closely related to the level sum of functions:

Theorem 3.1. For arbitrary extended real-valued functions $f, g: X \rightarrow \overline{\mathbb{R}}$ one always has

$$
(f \triangle g)^{c}=f^{c}+g^{c}
$$

Proof. An easy consequence of the definition (3.1) and of relations (2.2), $(2.5)$. 
Among many other interesting properties of the correspondence $f \longmapsto f^{c}$ we retain the following:

Proposition 3.2. Let $f: X \rightarrow \overline{\mathbb{R}}$ be a general extended real-valued function; the conjugate function $f^{c}$ satisfies the following properties:

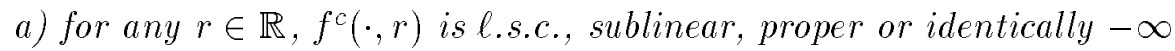

b) for any $y \in Y, f^{c}(y, \cdot)$ is l.s.c., nondecreasing.

\section{Moreover,}

$$
\left.\inf _{i \in I} f_{i}\right)^{c}=\sup _{i \in I} f_{i}^{c}
$$

for any family $\left(f_{i}\right)_{i \in I}$ of extended real-valued functions defined on $X$.

Proof. Let $r \in \mathbb{R}$; if $\{f<r\}=\emptyset$ then $f(\cdot, r)$ is identically $-\infty$; if $\{f<$ $r\} \neq \emptyset$ then $f(\cdot, r)$ is nothing but the support function of the nonvoid set

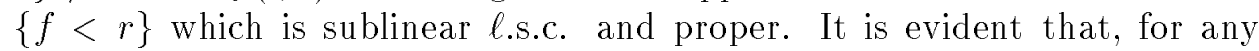
$y \in Y$, the function $f^{c}(y, \cdot)$ is nondecreasing, while the lower semicontinuity of this function follows from the easy but important property, valid for all $y \in Y, r, t \in \mathbb{R}:$

$$
f^{c}(y, r) \leq t \Longleftrightarrow \inf _{\langle x, y\rangle>t} f(x) \geq r
$$

On the other hand, (3.2) is a trivial consequence of the relation $\inf _{i \in I} f_{i}<$ $r\}=\bigcup_{i \in I}\left\{f_{i}<r\right\}$.

What formula (3.2) says is that the mapping $f \longmapsto f^{c}$ is a branch of a Galois correspondence $([9])$. Let us now describe the other branch.

Proposition 3.3. Let $f: X \rightarrow \overline{\mathbb{R}}$ and $\varphi: Y \times \mathbb{R} \rightarrow \overline{\mathbb{R}}$ be two extended real-valued functions. The following assertions are equivalent:

a) $f^{c}(y, r) \leq \varphi(y, r)$ for all $(y, r) \in Y \times \mathbb{R}$

b) $f(x) \geq \sup _{y \in Y} \sup \{r \in \mathbb{R}:\langle x, y\rangle>\varphi(y, r)\}$ for all $x \in X$.

Proof. By (3.3), a) amounts to saying that for any $x \in X, y \in Y, r \in \mathbb{R}$ one has

$$
\langle x, y\rangle>\varphi(y, r) \Longrightarrow f(x) \geq r
$$

or, equivalently, for any $x \in X, y \in Y$,

$$
f(x) \geq \sup \{r \in \mathbb{R}:\langle x, y\rangle>\varphi(y, r)\}
$$

that is exactly b).

Thus let us define the conjugate of an arbitrary extended real-valued function $\varphi: Y \times \mathbb{R} \rightarrow \overline{\mathbb{R}}$ by setting

$$
\varphi^{\gamma}(x):=\sup _{y \in Y} \sup \{r \in \mathbb{R}:\langle x, y\rangle>\varphi(y, r)\}
$$

for all $x \in X$. We then obtain the other branch of the Galois correspondence above mentioned and the related properties:

$$
\begin{aligned}
& f^{c} \leq \varphi \Longleftrightarrow f \geq \varphi^{\gamma}, \\
& \left(\inf _{i \in I} \varphi_{i}\right)^{\gamma}=\sup _{i \in I} \varphi_{i}^{\gamma},
\end{aligned}
$$


for any extended real-valued functions $f: X \rightarrow \overline{\mathbb{R}}, \varphi: Y \times \mathbb{R} \rightarrow \overline{\mathbb{R}}$, $\varphi_{i}: Y \times \mathbb{R} \rightarrow \overline{\mathbb{R}}(i \in I)$. Moreover the composite operators

$$
\begin{gathered}
f \in \overline{\mathbb{R}}^{X} \longmapsto f^{c \gamma}:=\left(f^{c}\right)^{\gamma} \in \overline{\mathbb{R}}^{X} \\
\varphi \in \overline{\mathbb{R}}^{Y \times \mathbb{R}} \longmapsto \varphi^{\gamma c}:=\left(\varphi^{\gamma}\right)^{c} \in \overline{\mathbb{R}}^{Y \times \mathbb{R}}
\end{gathered}
$$

are isotone:

$$
f \leq g \Longrightarrow f^{c \gamma} \leq g^{c \gamma}, \varphi \leq \psi \Longrightarrow \varphi^{\gamma c} \leq \psi^{\gamma c}
$$

contracting:

$$
f^{c \gamma} \leq f, \varphi^{\gamma c} \leq \varphi
$$

and idempotent:

$$
\left(f^{c \gamma}\right)^{c \gamma}=f^{c \gamma},\left(\varphi^{\gamma c}\right)^{\gamma c}=\varphi^{\gamma c} .
$$

More precisely one always has

$$
f^{c \gamma c}=f^{c}, \varphi^{\gamma c \gamma}=\varphi^{\gamma} .
$$

The class of functions $f \in \overline{\mathbb{R}}^{X}$ that coincide with their biconjugate $f^{c \gamma}$ (that we shall call regular in the sequel) is given by $\left\{\varphi^{\gamma}: \varphi \in \overline{\mathbb{R}}^{Y \times \mathbb{R}}\right\}=\left\{\varphi^{\gamma}: \varphi=\right.$ $\left.\varphi^{\gamma c}\right\}$. Of course a similar property holds for the class of regular functions $\varphi$ on $Y \times \mathbb{R}$.

The biconjugate of $f \in \overline{\mathbb{R}}^{X}$ (resp. $\varphi \in \overline{\mathbb{R}}^{Y \times \mathbb{R}}$ ) is nothing but the greatest regular minorant of $f$ (resp. $\varphi$ ):

$$
\begin{gathered}
f^{c \gamma}=\sup \left\{h \in \overline{\mathbb{R}}^{X}: h^{c \gamma}=h \text { and } h \leq f\right\} \\
\text { resp. } \varphi^{\gamma c}=\sup \left\{\xi \in \overline{\mathbb{R}}^{Y \times \mathbb{R}}: \xi^{\gamma c}=\xi \text { and } \xi \leq \varphi\right\} .
\end{gathered}
$$

All the above considerations are mere consequences of the theory of Galois correspondences $([5],[9])$. The description of the classes of regular functions is crucial for our purpose. We begin with regular functions on $X$.

TheOREM 3.4. Let $f: X \rightarrow \overline{\mathbb{R}}$ be an extended real-valued function. Then $f=f^{c \gamma}$ iff $f$ is l.s.c. quasiconvex.

Proof. It follows from (3.4) that for any $\varphi \in \overline{\mathbb{R}}^{Y \times \mathbb{R}}, t \in \mathbb{R}$,

$$
\left\{\varphi^{\gamma} \leq t\right\}=\bigcap_{y \in Y} \bigcap_{r>t}\{\langle\cdot, y\rangle \leq \varphi(y, r)\}
$$

that is an intersection of closed convex sets; hence the condition is necessary. Let us prove that it is also sufficient. Let $x \in X$ and $t \in \mathbb{R}$ be such that $r<f(x)$; thus $x$ does not belong to the closed convex set $\{f \leq r\}$; by the Hahn-Banach theorem there exists $y \in Y$ such that $\langle x, y\rangle>\sup \langle u, y\rangle \geq$ $f^{c}(y, r)$. By (3.4) we then have $f^{c \gamma}(x) \geq r$ so that, by taking the supremum over $r<f(x), f^{c \gamma}(x) \geq f(x)$. As the opposite inequality always holds the proof is complete.

Let us now describe the class of regular functions on $Y \times \mathbb{R}$.

Theorem 3.5. Let $\varphi: Y \times \mathbb{R} \rightarrow \overline{\mathbb{R}}$ be an extended real-valued function. Then, $\varphi=\varphi^{\gamma c}$ iff the following conditions are satisfied:

a) for any $r \in \mathbb{R}, \varphi(\cdot, r)$ is l.s.c., sublinear, proper or identically $-\infty$

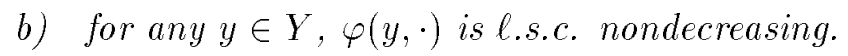


Proof. Necessity has been established in Proposition 3.2. Conversely, for all real number $r$ there exists a closed convex set $A_{r}$ such that $\varphi(\cdot, r)=$ $\sup \langle x, \cdot\rangle$; Let us set $f(x):=\inf \left\{t \in \mathbb{R}: x \in A_{t}\right\}$ and prove that $\varphi=f^{c}$. $x \in A_{r}$

First observe that $\{f<r\}=\bigcup_{t<r} A_{t}$. On the other hand, as $\varphi(y, \cdot)$ is l.s.c. nondecreasing,

$$
\sup _{t<r} \sup _{x \in A_{t}}\langle x, y\rangle=\sup _{x \in A_{r}}\langle x, y\rangle,
$$

so that

$$
\sup _{f(x)<r}\langle x, y\rangle=\sup \left(\langle x, y\rangle: x \in \bigcup_{t<r} A_{t}\right)=\sup _{x \in A_{r}}\langle x, y\rangle,
$$

that is to say $f^{c}=\varphi$.

\section{Explicit DUAL FORMUlas FOR THE LEVEL SUM OF L.S.C. QUASICONVEX FUNCTIONS.}

Thank to Theorem 3.1 and Theorem 3.4, let us observe that for any extended real-valued functions $f, g: X \rightarrow \overline{\mathbb{R}},\left(f^{c}+g^{c}\right)^{\gamma}$ is just the l.s.c. quasiconvex hull of the level sum $f \triangle g$. In the case when $f$ and $g$ are quasiconvex, $f \triangle g$ is quasiconvex too so that $\left(f^{c}+g^{c}\right)^{\gamma}$ is then the l.s.c. hull of $f \triangle g$. Now there exists a condition ensuring that the level sum of

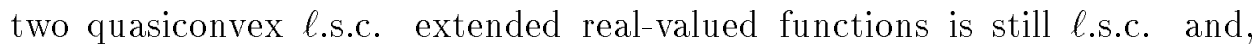
moreover, exact (i.e. the infimum is attained in (2.1)). This condition involves the so-called directions of majoration introduced by Amara ([1]). Let us briefly describe this notion. Given a quasiconvex function $f: X \rightarrow \mathbb{R}$, a vector $d \in X$ is said to be a direction of majoration of $f$ is there exists $\left(x_{0}, r_{0}\right) \in X \times \mathbb{R}$ such that

$$
f\left(x_{0}+t d\right) \leq r_{0} \text { for all } t \geq 0 .
$$

The set of directions of majoration of $f$ is a convex cone we denote by $C_{f}$. The function $f$ is called inf-locally compact if $\{f \leq r\}$ is locally compact for any $r \in \mathbb{R}$. We then have the following result:

Lemma 4.1. ([1]) Let $f, g: X \rightarrow \overline{\mathbb{R}}$ be two extended real-valued l.s.c. quasiconvex functions such that $C_{f} \cap-C_{g}=\{0\}$. Assume moreover that $f$ or $g$ is inf-locally compact. Then $f \triangle g$ is l.s.c., quasiconvex and exact.

It follows from the previous considerations that:

Theorem 4.2. For $f$ and $g$ as in Lemma 4.1 one has

$$
f \triangle g=\left(f^{c}+g^{c}\right)^{\gamma} \text {. }
$$

Clearly the dual formulations of the level sum we look for will come from the computation of the conjugate of the sum of two functions on $Y \times \mathbb{R}$. For doing this, the use of quasi-inverses is essential. Let us recall (see for instance [24]) that two nondecreasing functions $a, b: \mathbb{R} \rightarrow \overline{\mathbb{R}}$ are quasi-inverses if for all real numbers $r, s$ one has

$$
\begin{aligned}
& a(r)<s \Longrightarrow r \leq b(s) \\
& a(r)>s \Longrightarrow r \geq b(s) .
\end{aligned}
$$

ESAIM: COCV, SEPTEMBER 1998, Vol. 3, 329-343 
Among all the quasi-inverses of $a$ there is a smallest one namely

$$
a^{e}(s)=\sup \{r \in \mathbb{R}: a(r)<s\}=\inf \{r \in \mathbb{R}: a(r) \geq s\}
$$

which is also the only l.s.c. quasi-inverse of $a$. It is characterized by the relations

$$
a(r)<s \Longrightarrow r \leq a^{e}(s), r<a^{e}(s) \Longrightarrow a(r)<s
$$

for any real numbers $r, s$.

Given a function $f: X \rightarrow \overline{\mathbb{R}}$ and $y \in Y$, typical quasi-inverses are the following ([13], [24]): $p_{y}$ and $b_{y}, p_{y}$ and $\beta_{y}, \alpha_{y}$ and $b_{y}, \alpha_{y}$ and $\beta_{y}$ where $p_{y}(r)=$ $f^{c}(y, r), \alpha_{y}(r)=\sup _{f(x) \leq r}\langle x, y\rangle, \beta_{y}(s)=\inf _{\langle x, y\rangle>s} f(x), b_{y}(s)=\inf _{\langle x, y\rangle \geq s} f(x)$ $(r, s \in \mathbb{R})$.

Now observe that, from Theorem 4.2 and (3.4), (4.1), one has

$$
(f \triangle g)(x)=\sup _{y \in Y}\left(p_{y}+q_{y}\right)^{e}(\langle x, y\rangle)
$$

for all $x \in X$, with $p_{y}(r)=f^{c}(y, r)$ and $q_{y}(r)=g^{c}(y, r)$. Hence, to apply in concrete terms formula (4.2), we need to estimate the smallest quasi-inverse of the sum of two nondecreasing extended real-valued functions. In the next two lemmas $a_{1}$ and $a_{2}$ are such functions and we denote by $b_{i}$ a quasi-inverse of $a_{i}(i=1,2)$. We also set

$$
\left(b_{1} \nabla b_{2}\right)(s)=\sup _{s_{1}+s_{2}=s} b_{1}\left(s_{1}\right) \wedge b_{2}\left(s_{2}\right) \quad(s \in \mathbb{R}) .
$$

LeMma 4.3. $\left(a_{1}+a_{2}\right)^{e} \leq\left(b_{1} \nabla b_{2}\right) \vee \inf _{\mathbb{R}} b_{1} \vee \inf _{\mathbb{R}} b_{2}$.

Proof. Let $s \in \mathbb{R}$; observe that

$$
\left(a_{1}+a_{2}\right)^{e}(s)=\sup \left\{r \in \mathbb{R}: a_{1}(r) \dot{+} a_{2}(r)<s\right\} \vee r_{1} \vee r_{2}
$$

where $r_{i}=\sup \left\{r \in \mathbb{R}: a_{i}(r)=-\infty\right\}(i=1,2)$. In fact, one easily gets $r_{i}=$ $\inf _{\mathbb{R}} b_{i}(i=1,2)$. Now by $(2.6): a_{1}(r) \dot{+} a_{2}(r)<s \Longleftrightarrow \exists s_{1}, s_{2} \in \mathbb{R}: s_{1}+s_{2}=s$, $a_{i}(r)<s_{i}$; thus $r<b_{i}\left(s_{i}\right)(i=1,2)$; therefore, $\sup \left\{r \in \mathbb{R}: a_{1}(r) \dot{+} b_{1}(r)<\right.$ $s\} \leq\left(b_{1} \nabla b_{2}\right)(s)$ and Lemma 4.3 is proved.

The next relation is linked with a formula given in [22] (Lemma 2.4) for non-negative functions.

LEMMA 4.4. $\left(a_{1}+a_{2}\right)^{e} \leq b_{1} \triangle b_{2}$.

Proof. Let $\bar{r}>\left(b_{1} \triangle b_{2}\right)(s)$. Thus there exist $s_{1}, s_{2} \in \mathbb{R}$ such that $s_{1}+s_{2}=s$ and $b_{i}\left(s_{i}\right)<\bar{r}(i=1,2)$. We then have $s_{i} \leq a_{i}(\bar{r})$ so that $s \leq a_{1}(\bar{r})+a_{2}(\bar{r})$ and $\bar{r} \geq \inf \left\{r \in \mathbb{R}: a_{1}(r)+a_{2}(r) \geq s\right\}=\left(a_{1}+a_{2}\right)^{e}(s)$.

We are now ready to state the main result of this section:

TheOREM 4.5. Let $f, g: X \rightarrow \overline{\mathbb{R}}$ be two extended real-valued l.s.c. quasiconvex functions such that $C_{f} \cap-C_{g}=\{0\}$. Assume that $f$ or $g$ is inf-locally compact. Then for all $\bar{x} \in X$ :

$$
\begin{aligned}
(f \triangle g)(\bar{x}) & =\sup _{y \in Y} \sup _{s+t=\langle\bar{x}, y\rangle} \inf _{\langle u, y\rangle \geq s} f(u) \wedge \inf _{\langle v, y\rangle \geq t} g(v) \\
& =\sup _{y \in Y} \inf _{s+t=\langle\bar{x}, y\rangle} \inf _{\langle u, y\rangle \geq s} f(u) \vee \inf _{\langle v, y\rangle \geq t} g(v) .
\end{aligned}
$$


Proof. For each $y \in Y$ let us consider the quasi-inverse $b_{y}(s)=\inf _{\langle u, y\rangle \geq s} f(u)$ (resp. $\left.\quad c_{y}(s)=\inf _{\langle v, y\rangle \geq s} g(v)\right)$ of $p_{y}$ ( resp. $q_{y}$ ), and observe that $\inf _{\mathbb{R}} b_{y}=$ $\inf _{X} f, \inf _{\mathbb{R}} c_{y}=\inf _{X} g$. From Lemma 4.3, (4.2), and (2.4) we then have

$$
\begin{aligned}
(f \triangle g)(\bar{x}) & \leq \sup _{y \in Y}\left(b_{y} \nabla c_{y}\right)(\langle\bar{x}, y\rangle) \vee \inf _{X} f \vee \inf _{X} g \\
& \leq \sup _{y \in Y}\left(b_{y} \nabla c_{y}\right)(\langle\bar{x}, y\rangle) \vee \inf _{X}(f \triangle g) .
\end{aligned}
$$

Now observe that for any $y \in Y$

$$
\left(b_{y} \nabla c_{y}\right)(\langle\bar{x}, y\rangle) \leq(f \triangle g)(\bar{x}) .
$$

In fact let $\bar{s}, \bar{t} \in \mathbb{R}, \bar{s}+\bar{t}=\langle\bar{x}, y\rangle, \bar{u}, \bar{v} \in X, \bar{u}+\bar{v}=\bar{x}$. If $\langle\bar{u}, y\rangle \geq \bar{t}$ then

$$
f(\bar{u}) \vee g(\bar{v}) \geq \inf _{\langle u, y\rangle \geq \bar{t}} f(u) \geq \inf _{\langle u, y\rangle \geq \bar{t}} f(u) \wedge \inf _{\langle v, y\rangle \geq \bar{s}} g(v),
$$

If $\langle\bar{u}, y\rangle\langle\bar{t}$ then $\langle\bar{v}, y\rangle \geq \bar{s}$ and

$$
f(\bar{u}) \vee g(\bar{v}) \geq \inf _{\langle v, y\rangle \geq \bar{s}} g(v) \geq \inf _{\langle u, y\rangle \geq \bar{t}} f(u) \wedge \inf _{\langle v, y\rangle \geq \bar{s}} g(v),
$$

hence (4.6) holds. Now (2.3), (4.5) and (4.6) entail (4.3). Let us prove (4.4).

Lemma $4.4,(4.1)$ and (4.2) give the inequality $\leq$ in (4.4). Conversely, let $\bar{y} \in Y, \bar{u}, \bar{v} \in X, \bar{u}+\bar{v}=\bar{x} ;$ thus

$$
\begin{aligned}
\inf _{s+t=\langle\bar{x}, y\rangle} b_{y}(s) \vee c_{y}(s) & \leq b_{y}(\langle\bar{u}, y\rangle) \vee c_{y}(\langle\bar{v}, y\rangle) \\
& \leq f(\bar{u}) \vee g(\bar{v})
\end{aligned}
$$

so that the inequality $\geq$ in (4.4) is satisfied.

REMARK 4.6. A careful reading of the proof of Theorem 4.5 shows that with the same hypothesis one has also

$$
(f \triangle g)(\bar{x})=\sup _{y \in Y} \sup _{s+t=\langle\bar{x}, y\rangle} \inf _{\langle u, y\rangle>s} f(u) \wedge \inf _{\langle v, y\rangle \geq t} g(v) .
$$

Formula (4.7) is particularly interesting when $f$ is the valley function $v_{A}$ of a closed convex set $A \subset X\left(v_{A}(x)=-\infty\right.$ if $x \in A, v_{A}(x)=+\infty$ if $\left.x \in X \backslash A\right)$. In such a case

$$
\inf _{\langle u, y\rangle>s} v_{A}(u)=+\infty \quad \text { if } \quad i_{A}^{*}(y) \leq s,-\infty \quad \text { if } \quad i_{A}^{*}(y)>s .
$$

Denoting by $0^{+} A$ the recession cone of $A$ and $b(A):=\left\{i_{A}^{*}<+\infty\right\}$ the barrier cone of $A$ we then obtain from (4.7) a duality formula for the minimization of a quasiconvex function over a convex set.

Corollary 4.7. Let $A \subset X$ be a closed locally compact convex set, $g: X \rightarrow$ $\overline{\mathbb{R}}$ a l.s.c. quasiconvex function; assume that $0^{+} A \cap C_{g}=\{0\}$; then

$$
\min _{x \in A} g(x)=\sup _{y \in b(A)} \inf _{\langle v, y\rangle \leq i_{A}^{*}(y)} g(v) .
$$

More generally, Theorem 4.5 applies to the optimization problem

$$
\text { minimize } g(x) \vee h(x) \text { for } x \in X
$$

ESAIM: Cocv, September 1998, Vol. 3, 329-343 
where $g, h: X \rightarrow \overline{\mathbb{R}}$ are two extended real-valued l.s.c. quasiconvex functions. Setting $f(x):=h(-x)$ and taking $\bar{x}=0$ in Theorem 4.5 and (4.7) we get:

Corollary 4.8. Let $g, h: X \rightarrow \overline{\mathbb{R}}$ be two extended real-valued l.s.c. quasiconvex functions such that $C_{g} \cap C_{h}=\{0\}$. Assume that $g$ or $h$ is inf-locally compact; then

$$
\begin{aligned}
\min _{x \in X} g(x) \vee h(x) & =\sup _{\substack{y \in Y \\
t \in \mathbb{R}}} \inf _{\langle u, y\rangle \leq t} h(u) \wedge \inf _{\langle v, g\rangle \geq t} g(v) \\
& =\sup _{\substack{y \in Y \\
t \in \mathbb{R}}} \inf _{\langle u, y\rangle<t} h(u) \wedge \inf _{\langle v, g\rangle \geq t} g(v) \\
& =\sup _{y \in Y} \inf _{t \in \mathbb{R}\langle v, y\rangle \geq t} \inf _{\left\langle v(u) \vee \inf _{\langle v, y\rangle \leq t} g(v) .\right.}
\end{aligned}
$$

REMARK 4.9. It should be emphasized that formulas quite similar to (4.8) and (4.9) has been established under totally different assumptions in [35] where it is shown that they entail the usual convex duality formulas.

We conclude this section by describing the conjugates of an important class of quasiconvex functions, namely the radial quasiconvex functions. Let us consider a reflexive Banach space $X$ equipped with a norm || || and denote by \|\|$_{*}$ the dual norm: $\|y\|_{*}=\max _{\|x\|=1}\langle x, y\rangle$, for any $y$ in the topological dual $Y$ of $X$.

With any nondecreasing extended real-valued function $a:[0,+\infty[\rightarrow \mathbb{R}$ is associated the quasiconvex function $f=a \circ\|\|$. Setting $\tilde{a}(r)=a(0)$ for $r \in]-\infty, 0[$ and $\tilde{a}(r)=a(r)$ for $r \in[0,+\infty$ [ we get a nondecreasing function $\tilde{a}: \mathbb{R} \rightarrow \overline{\mathbb{R}}$ such that $\tilde{a}(0)=\inf _{\mathbb{R}} \tilde{a}$. We then have $\tilde{a}^{e}(s)=-\infty$ if $s \in$ ]$-\infty, a(0)] \cap \mathbb{R}, \tilde{a}^{e}(s) \geq 0$ if $\left.s \in\right] a(0),+\infty[$. Adopting the conventions

$$
(-\infty) \times 0=-\infty,(+\infty) \times 0=0,
$$

the quasiconjugate of $f=a \circ\|\|$ can then be described as follows:

Proposition 4.10. Let $a$ and $\tilde{a}$ be as above; then

$$
(a \circ\|\|)^{c}(y, s)=\tilde{a}^{e}(s)\|y\|_{*}
$$

for any $y \in Y, s \in \mathbb{R}$.

Proof. By (3.1) and (4.1) we get

$$
f^{c}(y, s)=\sup _{a(\|x\|)<s}\langle x, y\rangle \leq \sup _{\|x\| \leq \tilde{a}^{e}(s)}\langle x, y\rangle,
$$

so that, taking (4.11) into account,

$$
f^{c}(y, s) \leq \tilde{a}^{e}(s)\|y\|_{*}
$$

with equality for $y=0$.

Conversely assume that $y \neq 0$ and let $t \in \mathbb{R}$ be such that $t<\tilde{a}^{e}(s)\|y\|_{*} ;$ by (4.1): $\tilde{a}\left(t /\|y\|_{*}\right)<s$. If $t \leq 0$ then $s>a(0)$ and $f^{c}(y, s) \geq 0$; if $t>0$ 
let us choose $x \in X$ such that $\|x\|=1$ and $\langle x, y\rangle=\|y\|_{*}$; we then have $f^{c}(y, s) \geq t\langle x /\langle x, y\rangle, y\rangle=t$ so that in any case $f^{c}(y, s) \geq \tilde{a}^{e}(s)\|y\|_{*}$.

What Proposition 4.10 says is that the conjugate of a radial quasiconvex function can be written as the product of a nondecreasing function by the dual norm. Let us see how to compute the second conjugate of such functions.

Proposition 4.11. Let $\varphi: Y \times \mathbb{R} \rightarrow \overline{\mathbb{R}}$ be defined by

$$
\varphi(y, r)=b(r)\|y\|_{*}
$$

with $b: \mathbb{R} \rightarrow \overline{\mathbb{R}}$ nondecreasing; then,

$$
\varphi^{\gamma}=b^{e} \circ\|\|
$$

Proof. By (3.4) one has

$$
\varphi^{\gamma}(x)=\sup _{y \in Y} \sup \left(r \in \mathbb{R}: b(r)\|y\|_{*}<\langle x, y\rangle\right) .
$$

It follows from (4.11) that for $y=0$ one has:

$$
\sup \left(r \in \mathbb{R}: b(r)\|y\|_{*}\langle\langle x, y\rangle)=\sup (r \in \mathbb{R}: b(r)=-\infty)=\inf _{\mathbb{R}} b^{e} ;\right.
$$

thus,

$$
\begin{aligned}
\varphi^{\gamma}(x) & =\sup _{y \neq 0} \sup \left(r \in \mathbb{R}: b(r)<\langle x, y\rangle /\|y\|_{*}\right) \vee \inf _{\mathbb{R}} b^{e} \\
& =\sup _{y \neq 0} b^{e}\left(\langle x, y\rangle /\|y\|_{*}\right) .
\end{aligned}
$$

As $b^{e}$ is $\ell$.s.c. nondecreasing we then have $\varphi^{\gamma}(x)=b^{e}\left(\underset{y \neq 0}{\sup }\langle x, y\rangle /\|y\|_{*}\right)=$ $b^{e}(\|x\|)$.

Corollary 4.12. Let $f=a \circ\|\|$ with $a:[0,+\infty[\rightarrow \overline{\mathbb{R}}$ nondecreasing. Then $f^{c \gamma}=\bar{a} \circ\|\|$ where $\bar{a}$ is the l.s.c. hull of $a$.

Proof. By Proposition 4.10, $f^{c}(y, s)=\tilde{a}^{e}(s)\|y\|_{\star}$; setting $b=\tilde{a}^{e}, b^{e}$ is nothing but the l.s.c. hull $\overline{\tilde{a}}$ of $\tilde{a}$. By Proposition 4.11 we then have $f^{c \gamma}=$ $\overline{\tilde{a}} \circ\|\|=\bar{a} \circ\|\|$.

Corollary 4.13. Let $\varphi: Y \times \mathbb{R} \rightarrow \overline{\mathbb{R}}, \varphi(y, s)=b(s)\|y\|_{*}$ with $b: \mathbb{R} \rightarrow$ $[0,+\infty]$ nondecreasing; thus

$$
\varphi^{c \gamma}(y, s)=\bar{b}(s)\|y\|_{*}
$$

for any $y \in \mathbb{R}^{n}, s \in \mathbb{R}$.

Proof. By Proposition 4.11, $\varphi^{\gamma}=b^{e} \circ\|\|$; now let $a(r):=b^{e}(r)$ for $r \geq 0$; as $b$ is nonnegative one has $\tilde{a}=b^{e}$ and the result follows from Proposition 4.10 together with the relation $b^{e e}=\bar{b}$. 


\section{Application to Hamilton-Jacobi EQUATIONS}

In order to solve the Bellman equation in $L_{\infty}$ spaces, an explicit formula has been recently derived by Barron and al. ([7]) for the corresponding Hamilton-Jacobi equation

$$
\left.u_{t}=-H(D u, u) \quad \text { on } \quad\right] 0,+\infty\left[\times \mathbb{R}^{n} \quad \text { with } \quad u(0, x)=g(x) .\right.
$$

The hamiltonian $H(y, r)$ is here finite-valued, continuous, nondecreasing in $r$, sublinear in $y$, and the function $g$ Lipschitz continuous and bounded. Thus, the unique viscosity solution $([6],[10], \ldots)$ is given by

$$
u(t, x)=\inf _{z \in \mathbb{R}^{n}}\left(h\left(\frac{x-z}{t}\right) \vee g(z)\right)
$$

where $h$ is the quasiconvex function defined by

$$
h(x)=\inf \left\{r \in \mathbb{R}: H(y, r) \geq\langle x, y\rangle, \forall y \in \mathbb{R}^{n}\right\} .
$$

As $H(y, \cdot)$ is nondecreasing, observe that the function $h$ above is nothing but the $\gamma$-conjugate of $H$, namely:

$$
h(x)=H^{\gamma}(x)=\sup _{y \in \mathbb{R}^{n}} \sup \{r \in R: H(y, r)<\langle x, y\rangle\} .
$$

On the other hand, setting $h_{[t]}(x)=h\left(\frac{x}{t}\right)$ one can write

$$
u(t, x)=\left(h_{[t]} \triangle g\right)(x) .
$$

We are interested in the case when the initial condition $g$ is quasiconvex but not necessarily Lipschitz continuous or bounded (see [36]). Let us give some general properties inspired by the convex case (see e.g. [14] [26]).

LEMMA 5.1. Let $h: \mathbb{R}^{n} \rightarrow \overline{\mathbb{R}}$ be an extended real-valued quasiconvex function; then

$$
h_{[r+s]}=h_{[r]} \triangle h_{[s]}
$$

for all positive real numbers $r, s$.

Moreover the function $v$ defined on $] 0,+\infty\left[\times \mathbb{R}^{n}\right.$ by $v(t, x)=h_{[t]}(x)$ is jointly quasiconvex.

Proof. Let $x, x_{1}, x_{2} \in X$ with $x_{1}+x_{2}=x$; one has $\frac{x}{r+s}=\frac{r}{r+s}\left(\frac{x_{1}}{r}\right)+\frac{s}{r+s}\left(\frac{x_{2}}{s}\right)$ so that $h_{[r+s]}(x) \leq h_{[r]}\left(x_{1}\right) \vee h_{[s]}\left(x_{2}\right)$. This proves the inequality $\leq$ in $(5.3)$. Conversely,

$$
\left(h_{[r]} \triangle h_{[s]}\right)(x) \leq h_{[r]}\left(\frac{r}{r+s} x\right) \vee h_{[s]}\left(\frac{s}{r+s} x\right)=h_{[r+s]}(x) .
$$

We prove now that $v$ is jointly quasiconvex. Let $\left.x_{0}, x_{1} \in X, t_{0}, t_{1} \in\right] 0,+\infty[$ and $\lambda \in[0,1]$. One has

$$
x:=\frac{\lambda x_{1}+(1-\lambda) x_{0}}{\lambda t_{1}+(1-\lambda) t_{0}}=\frac{\lambda t_{1}}{\lambda t_{1}+(1-\lambda) t_{0}} \times \frac{x_{1}}{t_{1}}+\frac{(1-\lambda) t_{0}}{\lambda t_{1}+(1-\lambda) t_{0}} \times \frac{x_{0}}{t_{0}} ;
$$

therefore, $h(s) \leq h\left(\frac{x_{1}}{t_{1}}\right) \vee\left(\frac{x_{0}}{t_{0}}\right)$, that is $v\left(\lambda\left(t_{1}, x_{1}\right)+(1-\lambda)\left(t_{0}, x_{0}\right)\right) \leq$ $v\left(t_{1}, x_{1}\right) \vee v\left(t_{0}, x_{0}\right)$. 
Proposition 5.2. Let $g, h$ be two extended real-valued quasiconvex functions on $\mathbb{R}^{n}$; then the function $u$ defined in (5.2) is jointly quasiconvex on ] $0,+\infty\left[\times \mathbb{R}^{n}\right.$. If, moreover, $g$ and $h$ are $\ell . s . c$. and

$$
C_{g} \cap-C_{h}=\{0\}
$$

then $u$ is $\ell . s . c$. and exact.

Proof. One has $u(t, x)=\inf _{z \in \mathbb{R}^{n}}(v(t, z) \vee g(x-z))$; now the function $(t, x, z)$ $\longmapsto v(t, z) \vee g(x-z)$ is jointly quasiconvex. Taking the infimum on $z \in \mathbb{R}^{n}$ we obtain that $u$ is jointly quasiconvex (see e.g. [13]). Assume that (5.4) holds. Since $C_{h_{[t]}}=C_{h}$ for any $t>0$ one has $C_{g} \cap-C_{h_{[t]}}=\{0\}$ and $u$ is

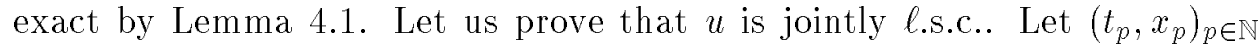
be a sequence with values in $\{u \leq r\}$ ( $r$ fixed in $\mathbb{R}$ ) converging to $(t, x)$. As $u$ is exact, there exist two sequences $\left(y_{p}\right)_{p \in \mathbb{N}}$ and $\left(z_{p}\right)_{p \in \mathbb{N}}$ such that $x_{p}=$ $t_{p} y_{p}+z_{p}, t_{p}>0, y_{p} \in\{h \leq r\}, z_{p} \in\{g \leq r\}$. Assume $\left(y_{p}\right)_{p \in \mathbb{N}}$ is unbounded: there exist $\varepsilon_{k} \stackrel{k \rightarrow \infty}{\rightarrow} 0_{+}$and a subsequence $\left(y_{p_{k}}\right)_{k \in \mathbb{N}}$ such that $\varepsilon_{k} y_{p_{k}} \stackrel{k \rightarrow \infty}{\rightarrow} y \neq 0$; it follows that $y \in C_{h}$ and $\varepsilon_{k} z_{p_{k}} \stackrel{k \rightarrow \infty}{\rightarrow}-t y \neq 0$; therefore, $y \in-C_{g}$ that is impossible. So, $\left(y_{p}\right)_{p \in \mathbb{N}}$ is bounded and, taking converging subsequences if necessary, we have $x_{p}=t_{p} y_{p}+z_{p} \stackrel{p \rightarrow \infty}{\rightarrow} x=t y+z$ with $y \in\{h \leq r\}$ and $z \in\{g \leq r\}$. Thus $u(t, x) \leq h(y) \vee h(z) \leq r$ and the proof is complete.

The description of the normal cone to a lower level set of $u$ is of crucial importance in the sequel. Recall that the normal cone of a convex subset $A \subset \mathbb{R}^{n}$ at a point $a \in A$ is the closed convex cone $N(A, a):=\left\{y \in \mathbb{R}^{n}\right.$ : $\sup \langle x, y\rangle \leq\langle a, y\rangle\}$. The following lemma points out that the normal cone of $x \in A$

a lower level set of a quasiconvex function includes the Frechet subdifferential of the function.

LEMMA 5.3. Let $f: \mathbb{R}^{n} \rightarrow \overline{\mathbb{R}}$ be an extended real-valued quasiconvex function, $a \in f^{-1}(\mathbb{R})$, and $y \in \mathbb{R}^{n}$ such that

$$
\liminf _{\|h\| \rightarrow 0} \frac{f(a+h)-f(a)-\langle h, y\rangle}{\|h\|} \geq 0 ;
$$

thus $y \in N(\{f \leq f(a)\}, a)$.

Proof. Let $f(a+h) \leq f(a)$ and $\varepsilon>0$; for $t>0$ small enough one has $f(a+t h)-f(a) \geq t(-\varepsilon+\langle h, y\rangle)$; as $f$ is quasiconvex it follows that

$$
0 \geq f(a) \vee f(a+h)-f(a) \geq t(-\varepsilon+\langle h, y\rangle) ;
$$

therefore, $\langle h, y\rangle \leq \varepsilon$ so that, as $\varepsilon>0$ is arbitrary, $y \in N(\{f \leq f(a)\}, a)$.

Now let us describe the normal cone of a lower level set of $u$.

LEMMA 5.4. Let $g$ and $h$ be two extended real-valued quasiconvex functions on $\mathbb{R}^{n}$ such that (5.4) holds, let $(\bar{t}, \bar{x}) \in u^{-1}(\mathbb{R}), \bar{r}=u(\bar{t}, \bar{x})$, and let $\bar{x}_{1}, \bar{x}_{2}$ in $\mathbb{R}^{n}$ be such that $\bar{x}=\bar{x}_{1}+\bar{x}_{2}, \bar{r}=h\left(\frac{\bar{x}_{1}}{\bar{t}}\right) \vee g\left(\bar{x}_{2}\right)$; thus $N(\{u \leq \bar{r}\},(\bar{t}, \bar{x}))$ is given by:

$$
\left\{\left(-\left\langle\frac{\bar{x}_{1}}{\bar{t}}, y\right\rangle, y\right): y \in N\left(\{h \leq \bar{r}\}, \frac{\bar{x}_{1}}{\bar{t}}\right) \cap N\left(\{g \leq \bar{r}\}, \bar{x}_{2}\right)\right\} .
$$


Proof. Let $(s, y) \in \mathbb{R} \times \mathbb{R}^{n}$; as the quasiconvex function $u$ is exact one has $(s, y) \in N(\{u \leq \bar{r}\},(\bar{t}, \bar{x}))$ iff

$$
s t+\left\langle x_{1}, y\right\rangle+\left\langle x_{2}, y\right\rangle \leq s \bar{t}+\left\langle\bar{x}_{1}, y\right\rangle+\left\langle\bar{x}_{2}, y\right\rangle
$$

for all $t>0, x_{1} \in t\{h \leq \bar{r}\}, x_{2} \in\{g \leq \bar{r}\}$.

Taking $t=\bar{t}$ and $x_{2}=\bar{x}_{2}$ we get $y \in N\left(\{h \leq \bar{r}\}, \frac{\bar{x}_{1}}{\bar{t}}\right)$.

Taking $t=\bar{t}$ and $x_{1}=\bar{x}_{1}$ we get $y \in N\left(\{g \leq \bar{r}\}, \bar{x}_{2}\right)$.

Taking $x_{1}=\frac{t}{\bar{t}} \bar{x}_{1}$ and $x_{2}=\bar{x}_{2}$ we get $s=-\left\langle\frac{\bar{x}_{1}}{\bar{t}}, y\right\rangle$.

We then have the inclusion $\subset$ in the announced formula; the other inclusion being obvious, the proof is complete.

We are now in a position to state the main result of this section saying that if the initial condition $g$ is continuous and quasiconvex then the function $u$ in (5.2) is still a viscosity solution of (5.1). More precisely:

TheOREM 5.5. Let $H$ be as in the beginning of this section, $h=H^{\gamma}$, and $g$ : $\mathbb{R}^{n} \rightarrow \mathbb{R}$ a continuous quasiconvex function such that $h(0) \leq \inf _{\mathbb{R}^{n}} g$. Thus, the continuous quasiconvex function $u(t, x)=\left(h_{[t]} \triangle g\right)(x)$ is a viscosity solution of (5.1).

Proof. By Theorem 3.5 one has $H=h^{c}$; as $H$ is finite valued the sets $\{h<r\}$ are bounded so that $C_{h}=\{0\}$. It then follows from Proposition 5.2 that $u$ is $\ell . s . c$. quasiconvex and exact; as $g$ is real-valued, it ensues that $u$ is real-valued too. Moreover $u(t, x)=\inf _{y \in \mathbb{R}^{n}}(h(y) \vee g(x-t y))$ so that $u$ is upper semicontinuous (for each $y \in \mathbb{R}^{n}$, the function $\left.(t, x) \in\right] 0,+\infty\left[\times \mathbb{R}^{n} \longmapsto\right.$ $h(y) \vee g(x-t y)$ is continuous). Hence $u$ is continuous with real values. Let us check that $u$ is a viscosity subsolution ot (5.1). On this point we follow the proof of [7], Theorem 3.1: let $\varphi:] 0,+\infty\left[\times \mathbb{R}^{n} \rightarrow \mathbb{R}\right.$ be a differentiable function such that $u-\varphi$ has a local maximum at $\left(t_{0}, x_{0}\right)$. We have to prove that $\varphi_{t}\left(t_{0}, x_{0}\right) \leq-H\left(D_{x} \varphi\left(t_{0}, x_{0}\right), u\left(t_{0}, x_{0}\right)\right)$. By Theorem 3.5 this amounts to saying that, given $x$ such that $h(x)<u\left(t_{0}, x_{0}\right)$, we have

$$
\varphi_{t}\left(t_{0}, x_{0}\right) \leq-\left\langle x, D_{x} \varphi\left(t_{0}, x_{0}\right)\right\rangle .
$$

Now, for $\sigma>0$ small enough one has

$$
\frac{\varphi\left(t_{0}-\sigma, x_{0}-\sigma x\right)-\varphi\left(t_{0}, x_{0}\right)}{-\sigma} \leq \frac{u\left(t_{0}-\sigma, x_{0}-\sigma x\right)-u\left(t_{0}, x_{0}\right)}{-\sigma} .
$$

Taking Lemma 5.1 into account we get $u\left(t_{0}, x_{0}\right) \leq u\left(t_{0}-\sigma, x_{0}-\sigma x\right) \vee h(x)$ so that $\frac{\varphi\left(t_{0}-\sigma, x_{0}-\sigma x\right)-\varphi\left(t_{0}, x_{0}\right)}{-\sigma} \leq 0$. Finally, sending $\sigma \rightarrow 0^{+}$one obtains (5.5).

We prove now that $u$ is a supersolution of (5.1). Let $(\bar{t}, \bar{x})$ be a local minimum of $u-\varphi$; for $\|(t-\bar{t}, x-\bar{x})\|$ small enough we then have $u(t, x)-u(\bar{t}, \bar{x}) \geq \varphi(t, x)-\varphi(\bar{t}, \bar{x})$

$=(t-\bar{t}) \varphi_{t}(\bar{t}, \bar{x})+\left\langle x-\bar{x}, D_{x} \varphi(\bar{t}, \bar{x})\right\rangle+\varepsilon(t, x)\|(t-\bar{t}, x-\bar{x})\|$ with $\lim _{(t, x) \rightarrow(\bar{t}, \bar{x})} \varepsilon(t, x)=0$. 
Thus,

$$
\liminf _{(t, x) \rightarrow(\bar{t}, \bar{x})} \frac{u(t, x)-u(\bar{t}, \bar{x})-(t-\bar{t}) \varphi_{t}(\bar{t}, \bar{x})-\left\langle x-\bar{x}, D_{x} \varphi(\bar{t}, \bar{x})\right\rangle}{\left\|\left(t-t_{0}, x-x_{0}\right)\right\|} \geq 0 .
$$

Now, $u$ being jointly quasiconvex, Lemma 5.3 says that

$$
\left(\varphi_{t}(\bar{t}, \bar{x}), D_{x} \varphi(\bar{t}, \bar{x})\right) \in N(\{u \leq u(\bar{t}, \bar{x})\},(\bar{t}, \bar{x})) .
$$

As $u$ is exact (Proposition 5.2) it follows from Lemma 5.4 that

$$
\varphi_{t}(\bar{t}, \bar{x})=-\left\langle\frac{\bar{x}_{1}}{\bar{t}}, D_{x} \varphi(\bar{t}, \bar{x})\right\rangle
$$

with $h\left(\frac{\bar{x}_{1}}{\bar{t}}\right) \leq u(\bar{t}, \bar{x})$. On the other hand the continuity of $H$ entails $H(y, r) \stackrel{t}{=} h^{c}(y, r)=\sup _{h(x)<r}\langle x, y\rangle=\sup _{h(x) \leq r}\langle x, y\rangle$ for all $y \in \mathbb{R}^{n}, r \in \mathbb{R}$, so that

$$
\varphi_{t}(\bar{t}, \bar{x}) \geq-H\left(D_{x} \varphi(\bar{t}, \bar{x}), u(\bar{t}, \bar{x})\right) .
$$

To conclude the proof let us verify the initial condition in (5.1). Let $\bar{x} \in X$ and $f^{t}(x):=h_{[t]}(x) \vee g(\bar{x}-x)$. As $h(0) \leq \inf _{R^{n}} g$ and $h$ is quasiconvex it follows that $\left(f^{t}\right)_{t>0}$ is a nonincreasing family. Since $h$ is coercive, $\lim _{t \rightarrow 0_{+}} h_{[t]}(x)=$ $+\infty$ if $x \neq 0, h(0)$ if $x=0$, so that $\left(f^{t}\right)_{0<t \leq 1}$ is a nonincreasing family of equicoercive l.s.c. functions with $\lim _{t \rightarrow 0_{+}} f^{t}(x)=+\infty$ if $x \neq 0, g(\bar{x})$ if $x=0$. Therefore, $u(0, \bar{x})=\lim _{t \rightarrow 0_{+}} \inf _{\mathbb{R}^{n}} f^{t}=\inf _{\mathbb{R}^{n}} \lim _{t \rightarrow 0_{+}} f^{t}=g(\bar{x})$.

REMARK 5.6. The condition $h(0) \leq \inf _{R^{n}} g$ in Theorem 5.5 is not very stringent; it is in particular satisfied when the lagrangian $H$ is nonnegative: in such a case one has $h(0)=-\infty$.

\section{REFERENCES}

[1] C. Amara: Directions de majoration d'une fonction quasiconvexe et applications, Serdica Math. J., 23, 1997, 1001-1018.

[2] H. Attouch, R.Wets: Epigraphical analysis, in Analyse non linéaire, H. Attouch, J.-P. Aubin, F. Clarke, I. Ekeland eds., Gauthier-Villars, Paris, 1989, 73-100.

[3] H. Attouch, M. Volle: Cutting and scanning methods in set-valued analysis, SetValued Analysis, 4, 1996, 135-155.

[4] M. Avriel, W. Diewert, S. Schaible, I. Zang: Generalized concavity, Plenum Press, New York, 1987.

[5] F. Baccelli, G. Cohen, G.J. Olsder, J.-P. Quadrat: Synchronization and linearity: an algebra for discrete event systems, John Wiley and Sons, New York, 1992.

[6] G. Barles: Solutions de viscosité des équations de Hamilton-Jacobi, Math. et Appl. 17, Springer-Verlag, 1994.

[7] E.N. Barron, R. Jensen, W. Liu: Hopf-Lax-Type Formula for $u_{t}+H\left(u, D_{u}\right)=0$, J. Diff. Eq., 126, 1996, 48-61.

[8] E.N. Barron, W. Liu: Calculus of variations in $L^{\infty}$, Appl. Math. Optim., 35, 1997, 237-263.

[9] G. Birkhoff: Lattice Theory, Providence, A.M.S., 1966.

[10] M.G. Crandall, P.-L. Lions: Viscosity solutions of Hamilton Jacobi equations, Trans. Amer. Math. Soc., 277, 1983, 1-42.

[11] J.-P. Crouzeix: Polaires quasiconvexes et dualité, C. R. Acad. Sci. Paris, 297, Série A, 1974, 955-958.

[12] J.-P. Crouzeix: Deux notions de polarité pour les fonctions quasiconvexes, $C . R$. Acad. Sci. Paris, 282, Série A, 1976, 107-109.

ESAIM: COCV, September 1998, Vol. 3, 329-343 
[13] J.-P. Crouzeix: Contributions à l'étude des fonctions quasiconvexes, Thesis, University of Clermont-Ferrand II, France, 1977.

[14] L.C. Evans: Some min-max methods for the Hamilton-Jacobi equation, Ind. Math. J., 33-1, 1984, 31-50.

[15] W. Fenchel: Convex cones, sets and functions, University of Princeton, 1953.

[16] M. Gondran: Analyse minimax, C. R. Acad. Sci. Paris, 323, Série I, 1996, 1249-1252.

[17] L. Hörmander: Sur la fonction d'appui des ensembles convexes dans un espace localement convexe, Ark. Mat., 3, 1954, 181-186.

[18] D.T. Luc, M. Volle: Level set under infimal convolution and level addition, J.O.T.A., 94-3, 1997, 695-714.

[19] J.E. Martinez-Legaz: Quasiconvex duality theory by generalized conjugations methods, Optimization, 19, 1988, 603-652.

[20] J.E. Martinez-Legaz, I. Singer: $\vee$-Dualities and $\perp$-dualities, Optimization, 22-4, 1991, 483-511.

[21] J.-J. Moreau: Inf-convolution, sous-additivité, convexité des fonctions numériques, $J$. Math. Pures Appl., 49, 1970, 109-154.

[22] J.-P. Penot: Starshaped functions and conjugacy, Preprint, University of Pau, France, 1996.

[23] J.-P. Penot, M. Volle: Another duality scheme for quasiconvex problems, International Series of Numerical Math., 84, 1988, Birkaüser Verlag Basel, 259-275.

[24] J.-P. Penot, M. Volle: Inversion of real-valued functions and applications, ZOR Methods and Models of Operations Research, 34, 1990, 117-141.

[25] J.-P. Penot, M. Volle: On quasiconvex duality, Math. Oper. Res., 15-4, 1990, $597-$ 625.

[26] P. Plazanet: Contributions à l'analyse des fonctions convexes et des différences de fonctions convexes. Application à l'optimisation et à la théorie des e.d.p., Thesis, University Paul Sabatier, Toulouse, France, 1990.

[27] R.T. Rockafellar: Duality theorems for convex functions, Bull. Amer. Math. Soc., 70, 1964, 189-192.

[28] R.T. Rockafellar: Convex Analysis, Princeton, 1970.

[29] A.M. Rubinov, B.M. Glower: On generalized quasiconvex conjugation, Contemp. Math., 204, 1997, 199-216.

[30] A. Seeger, M. Volle: On a convolution operation obtained by adding level sets: classical and new results, Operations Research, 29-2, 1995, 131-154.

[31] I. Singer: The lower semicontinuous quasiconvex hull as a normalized second conjugate, Nonlinear Anal. Theory Methods Appl., 7, 1983, 1115-1121.

[32] P.T. Thach: A nonconvex duality with a zero duality gap and applications, SIAM J. Optim., 4, 1994, 44-64.

[33] S. Traoré, M. Volle: On the level sum of two convex functions on Banach spaces, Journal of Convex Analysis, Part II of the Special Issues in Honor of R.T. Rockafellar, 3-1, 1996, 141-151.

[34] M. Volle: Conjugaison par tranches, Ann. Mat. Pura ed Appl., 139, 1985, 279-311.

[35] M. Volle: Quasiconvex duality for the max of two functions, Lecture Notes in Economics and Math. Systems, 452, 1997, 365-379.

[36] M. Volle: Conditions initiales quasiconvexes dans les équations de Hamilton-Jacobi, C. R. Acad. Sci. Paris, 325, Série I, 1997, 167-170. 